\title{
The Florida Barred Galaxy Program
}

\author{
J. H. Hunter, Jr. and S. T. Gottesman \\ University of Florida, Astronomy Department, P.O. Box 112055 \\ Gainesville, FL 32611-2055, U.S.A.
}

\begin{abstract}
For more than a decade, a group at the University of Florida has been constructing models of barred spiral galaxies that we have observed at the VLA. Group members include: S. T. Gottesman, G. Contopoulos, J. H. Hunter, several Ph.D. students, and collaborators at other institutions. The HI distributions in our galaxies are either gas rich, or nearly devoid of gas, in their bar regions. Initially, we deduced density distributions and potentials of the stellar disks and bars from near infrared observations, modeled the gas response assuming different pattern speeds, etc., and compared the models with our HI observations. This simple approach is successful in some cases, such as NGC 3359, but less successful in others. More recently, our approach has been toward selfconsistent models, including stars and gas. Our techniques range from the Contopoulos-Grosbøl-Kaufmann stellar dynamical approach to methods exploiting grid codes and SPH tree codes. Some of our observations and models will be reviewed, and some new results will be presented.
\end{abstract}

\section{Observations}

The galaxy group at the University of Florida has observed nine barred spirals at the $21 \mathrm{~cm}$ wavelength of atomic hydrogen (for references, see Gottesman 1992). The galaxies were observed at the VLA telescope of the NRAO. With the exception of one system, the angular resolution achieved was about $20^{\prime \prime}$, and (excepting three systems) the velocity resolution was $25 \mathrm{~km} \mathrm{~s}^{-1}$. (The exceptions are NGC 7479, angular resolution 6"; NGC 1073, NGC 3319 and NGC 7479, velocity resolution $12.6 \mathrm{~km} \mathrm{~s}^{-1}$.)

The galaxies were chosen primarily because of size and brightness, in order that their structure be well resolved and their features be recorded at high signal-to-noise. In addition, the systems were required to be isolated from other galaxies of comparable dimension. Furthermore, $R$ and $I$ band photometry were available (R. Buta, D. and B. Elmegreen, P. Grosbøl, and C. Telesco; private communications), thereby providing some information about the underlying stellar distributions.

Table 1 lists the objects. It can be seen that our survey includes a wide range of morphological types with some variation in luminosity class. Also, systems with and without rings are included.

One of the primary findings concerns the form of the large scale distribution of the HI, which Hunter (1990) has previously described as Class I and Class 
Table 1. Galaxies observed by the Florida group.

\begin{tabular}{clcc}
\hline Galaxy & Type & Diameter & $\sigma_{\max }(H I) / \sigma_{\text {center }}(H I)$ \\
\hline NGC 1398 & SBab(r) I & $6.6^{\prime}$ & 2.9 \\
NGC 3992 & SBb(rs) I & $7.6^{\prime}$ & 5.0 \\
NGC 1300 & SBb(s) I.2 & $6.5^{\prime}$ & 1.9 \\
NGC 1784 & SBbc(rs) I-II & $4.2^{\prime}$ & \\
NGC 7479 & SBbc(s) I-II & $4.4^{\prime}$ & 1.2 \\
NGC 3359 & SBc(s) I.8 & $6.3^{\prime}$ & 1.4 \\
NGC 1073 & SBc(rs) II & $4.9^{\prime}$ & 4.9 \\
NGC 3319 & SBcd(s) II.4 & $6.7^{\prime}$ & 1.0 \\
NGC 4731 & SBcd(s) III & $6.5^{\prime}$ & 1.0 \\
\hline
\end{tabular}

II systems. Class I objects have a very significant depletion in their central HI, while those of Class II show little or no central void and may have a bar rich in atomic hydrogen. In Figures 1 and 2 the HI distributions of two examples are shown, NGC 1398 (Class I) and NGC 3319 (Class II) - more details on these systems are given by Moore \& Gottesman (these proceedings). NGC 7479 is an interesting variant, for it has a central void in HI but has a bar that is rich in $H_{2}$ (see the paper by Laine \& Gottesman, these proceedings).
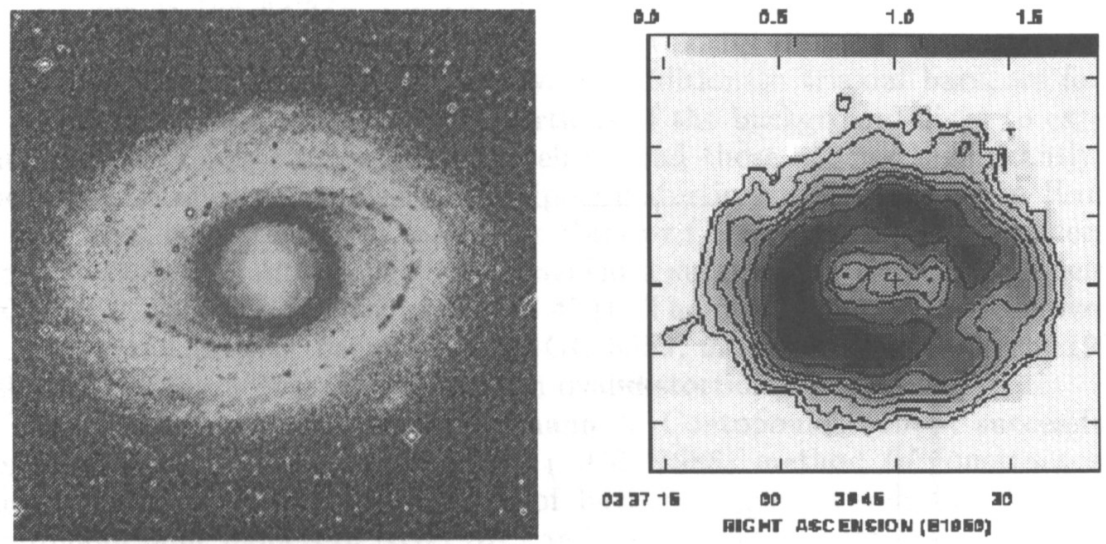

Figure 1. a) An optical image of NGC 1398, showing the inner ring.

b) The HI distribution in NGC 1398 .

In Figure 3 we compare the ratio of the value of the $\mathrm{HI}$ at its maximum to the HI value at the center, relative to the morphological type. A clear trend can be seen. Early types tend toward a paucity of central HI, while late types are rich in central HI and have gaseous (HI) bars. Furthermore, the central $H_{2}$ density for the class I system NGC 3992 cannot exceed that of the HI. There is a void in the total gas distribution. In addition, the one galaxy with a clear inner ring (NGC 1398) is the earliest system in our survey; undoubtedly this 

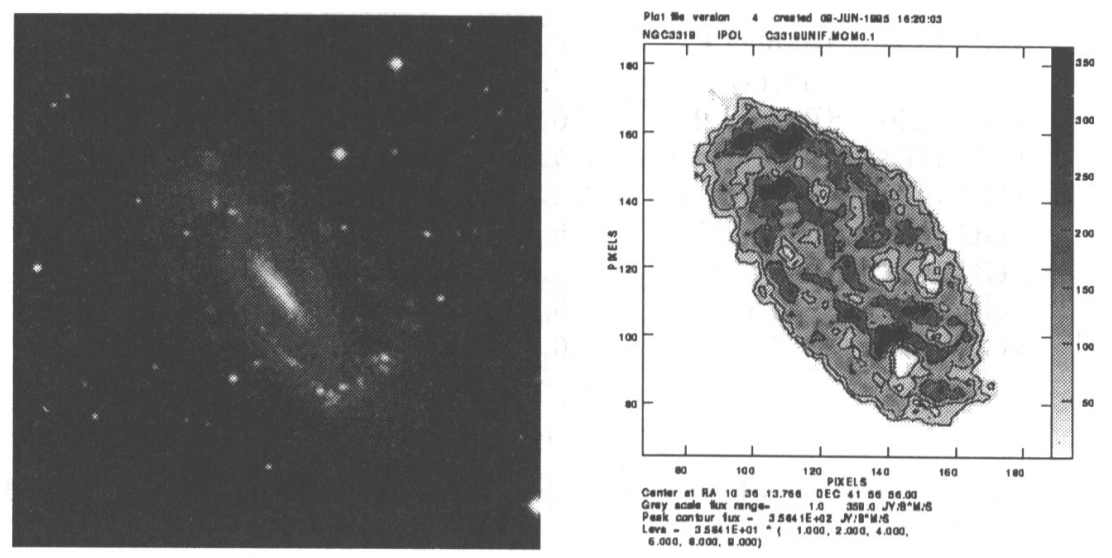

Figure 2. a) An optical image of NGC 3319. b) The HI distribution in NGC 3319.

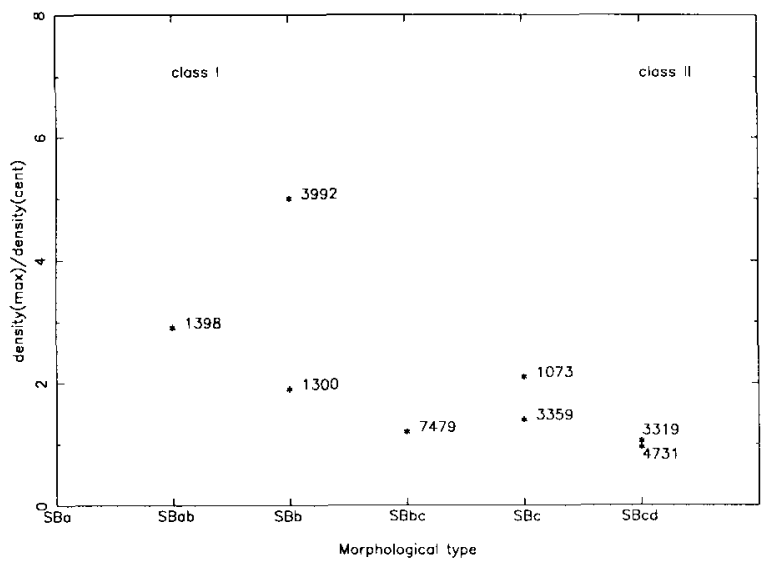

Figure 3. A comparison of the morphological type and the ratio of the maximum to central HI surface density in our program galaxies. 
is part of the same Class II to Class I morphological trend. Hunter's initial distinction represents only the extremes of this distribution. Finally, we voice a note of caution concerning these data. Although the angular resolutions are comparable for the material plotted in Figure 3, the linear resolutions are not uniform. While this may alter the details of the diagram, it will not remove the trend that can be seen; indeed, it may accentuate it.

These data are consistent with recent hypotheses regarding the dynamical evolution of galactic forms. Martinet (1995) has suggested that galaxies begin as slowly rotating, weakly concentrated objects that evolve dynamically into rapidly rotating more centrally condensed systems. Bars play an essential role in this redistribution of angular momentum. The abundant gas in late type galaxies loses angular momentum as it experiences the torque of the bar, and it falls toward the center of the galaxy. The ensuing evolution may change a. galaxy's morphology to earlier forms and it is possible for the growing central concentration of mass to destroy the stellar orbits that support the bar. A nonbarred, early type system may develop. Nothing in Figure 3 contradicts this scenario.

Our numerical modeling of barred spiral galaxies has proceeded along the following directions:

1) From optical, infrared and HI $21 \mathrm{~cm}$ data for a particular galaxy, we deduce mass distributions for the background disk, bar, etc. that are consistent with the observations, assign a pattern speed, $\Omega_{p}$, to the model bar and calculate the response of a massless gaseous disk component. When viewed at the same orientation as the galaxy, the gas in a successful model, having the correct pattern speed $\Omega_{p}$, will have the same radial velocity distribution and gaseous surface density as the observed galaxy. In addition to triaxial bars, we found it necessary to include oval $(2 \theta)$ distortions of the background disks to extend the nonaxisymmetric forces to radii well beyond those affected significantly by the bars. Detailed discussions of multipole distortions may be found in Hunter et al. (1988, hereafter H88), Sanders \& Huntley (1976) and Huntley, Sanders \& Roberts (1978). Hunter (1990) has provided a summary of our early models of NGC 1073, 1300, 3359, 3992 and NGC 4731. The most successful model overall is that of the gas-rich barred spiral NGC 3359, modeled by Ball $(1986,1992)$ using essentially a Toomre disk and an oval distortion.

2) Kaufmann (1993) and Kaufmann \& Contopoulos (1995) successfully generalized the Contopoulos-Grosbøl $(1986,1988)$ method to construct selfconsistent stellar dynamical models of barred spirals. Using this approach, Kaufmann built models of NGC 1073 and NGC 3992 (Figure 4).

3) We have used SPH tree codes, obtained from Hernquist and Heller and Shlosman, to simulate fully self-consistent models of barred spirals, including both stars and gas. Also, we have a Cartesian grid code, developed by E. Moore (1992) and a polar grid code (Kaufmann, private communication) that can be used for this purpose. While it is relatively easy to construct plausible generic models with these codes, as yet we have no successful systematic way of selecting initial conditions that will result in final models that match particular program galaxies. Also, star formation as well as supernovae and stellar winds must be included in a physically reasonable, but practically implementable, fashion. Further work is continuing on this subject. 


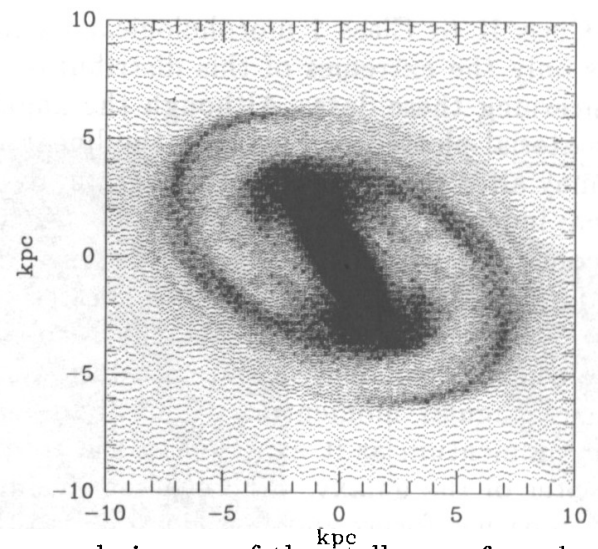

Figure 4. Grayscale image of the stellar surface density in the best model of NGC 3992 projected to galaxy's actual orientation.
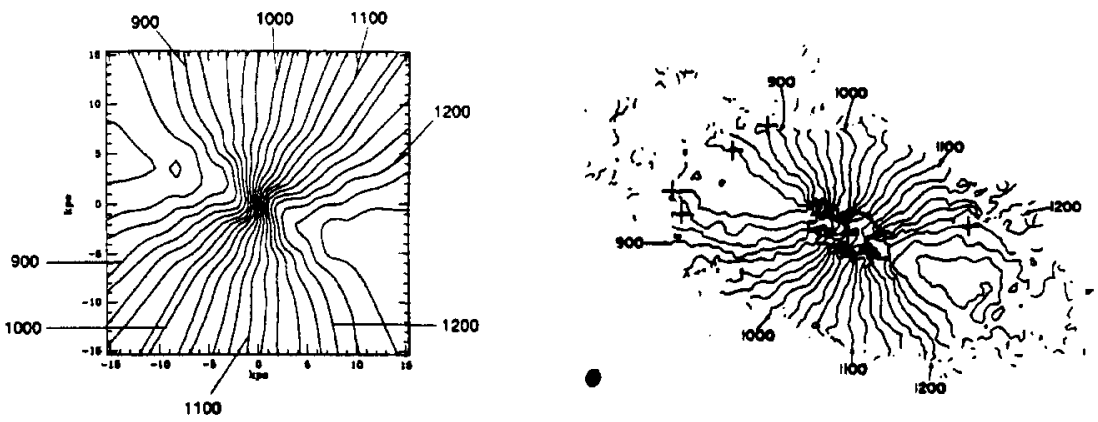

Figure 5. a) Gas velocity isopleths predicted from a self-consistent model of NGC 3992. b) Observed velocity isopleths in NGC 3992.

Gas in Kaufmann's model of NGC 3992 is shown in Figure 5. The match of his predicted radial velocity isopleths of the gas with the observed ones is impressive. However, contrary to observations, his calculated gaseous surface densities are large in the bar region. Indeed, the best model of NGC 3992 from H88 (Model 3, pp. 731-733) gives much better agreement with the observed surface density distribution, and its radial velocity isopleths match the observations quite well. Thus, as a practical matter approach 1) "works", even though the models are not self-consistent. Moreover, the amplitudes of the oval distortions in successful models of this type might be observable if they exist - for Model 3 , the maximum perturbed surface density is $34 \%$ of the unperturbed surface density at $\mathbf{r}=1.86 \mathrm{kpc}$. Our efforts in the near future will be directed toward combining approaches 2 and 3 above. Interestingly, all of our "successful" models, however they are constructed, have pattern speeds that place corotation slightly beyond the ends of their bars.

Hunter and Contopoulos have been collaborating with M. N. England on the behavior of vortices that are apparent in the gas when viewed in rotating 

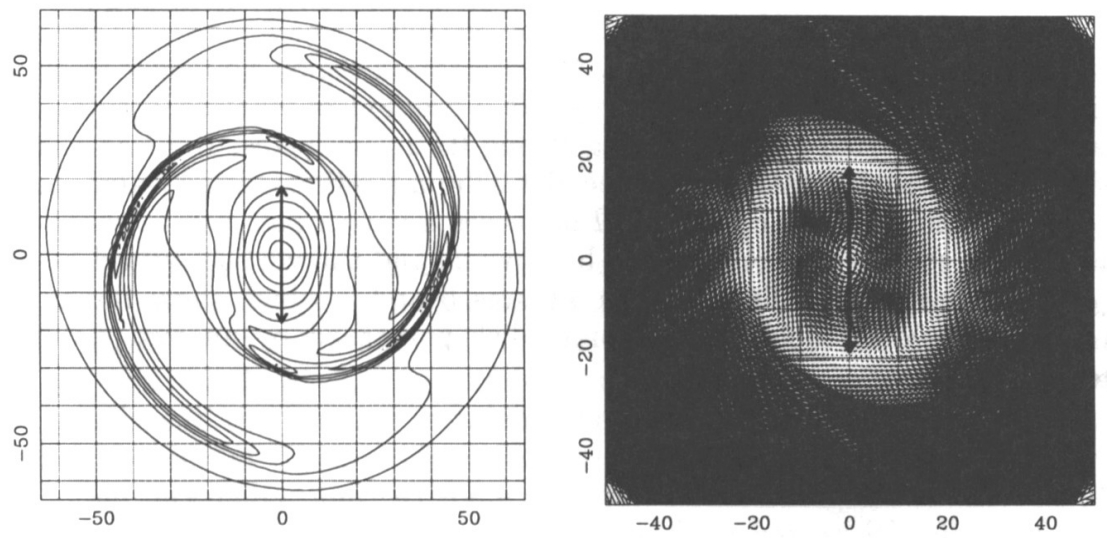

Figure 6 . a) The gaseous surface density of an $\mathrm{n}=0$ Toomre disk, excited by an oval distortion; $\Omega=\Omega_{p}$ and the corotation radius $=4.3$ kpc. b) The velocity field of the gas; stationary vortices located at $L_{4,5}$.

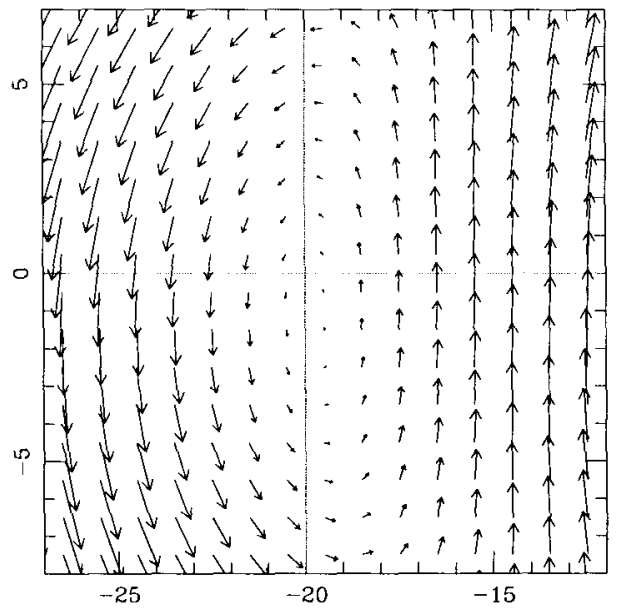

Figure 7. An enlargement of one of the vortices in Figure $6 \mathrm{~b}$. 
frames. When viewed in the bar frame with optimal $\Omega_{p}$, vortex pairs are located near $L_{4,5}$ and the circular corotation region is obvious. If the angular speed of the bar, $\Omega$, is increased (or decreased), the vortices persist at a different radius, and the corotation region becomes elliptical for relatively small variations from $\Omega_{p}$, but when $\Omega$ is very different from $\Omega_{p}$ the corotation region no longer resembles a circular annulus. This behavior suggests a method of locating corotation in relatively large barred galaxies having strong HI $21 \mathrm{~cm}$ fluxes. If a well resolved velocity field of such a galaxy would be viewed in a rigidly rotating frame, having its origin at the galaxy's center, as $\Omega$ increases a vortex pair will appear; when $\Omega=\Omega_{p}$, the corotation circle should be obvious and the vortices would be near $L_{4,5}$. This behavior is illustrated in Figures 6 and 7 which show the surface density and velocity fields of a Toomre disk perturbed by an oval distortion when $\Omega=\Omega_{p}$.

\section{References}

Ball, R. 1984, Ph.D. Dissertation, University of Florida

Ball, R. 1986, ApJ, 307, 453

Ball, R. 1992, ApJ, 395, 418

Contopoulos, G. \& Grosbøl, P. 1986, A\&A, 155, 11

Contopoulos, G. \& Grosbøl, P. 1988, A\&A, 197, 83

Gottesman, S. T. 1992, in Astrophysical Disks, S. F. Dermott, J. H. Hunter, Jr., \& R. E. Wilson, Annals of the New York Academy of Sciences, 675, 108

Hunter, J. H. Jr. 1990, in Galactic Models, J. R. Buchler, S. T. Gottesman, \& J. H. Hunter, Jr., Annals of the New York Academy of Sciences, 596, 174

Hunter, J. H. Jr., Ball, R., Huntley, J. M., England, M. N., \& Gottesman, S. T. 1988, ApJ, 324, 721

Huntley, J. M., Sanders, R. H., \& Roberts, W. W. 1978, ApJ, 221, 521

Kaufmann, D. E. 1993, Ph.D. Dissertation, University of Florida

Kaufmann, D. E. \& Contopoulos, G. 1995, to appear in A\&A

Martinet, L. 1995, Fundamentals of Cosmic Physics, 15, 341

Moore E. M. 1992, Ph.D. Dissertation, University of Florida

Sanders, R. H. \& Huntley, J. M. 1976, ApJ, 209, 53 